\title{
Subtipos moleculares del cáncer de mama: implicaciones pronósticas y características clínicas e inmunohistoquímicas
}

\section{Molecular subtypes of breast cancer: prognostic implications and clinical and immunohistochemical characteristics}

\author{
M.A. Arrechea Irigoyen ${ }^{1}$, F. Vicente García ${ }^{2}$, A. Córdoba Iturriagagoitia ${ }^{3}$, \\ B. Ibáñez Beroiz ${ }^{4}$, M. Santamaría Martínez ${ }^{3}$, F. Guillén Grima ${ }^{5}$
}

\section{RESUMEN}

Fundamento. Los carcinomas de mama representan un grupo heterogéneo de tumores, tanto en su comportamiento clínico como pronóstico. El objetivo del presente trabajo es clasificar los carcinomas de mama en subtipos moleculares mediante marcadores inmunohistoquímicos y analizar las características clinicopatológicas e inmunohistoquímicas y los patrones de supervivencia y recaída de los distintos subtipos.

Material y métodos. Se han clasificado 272 pacientes con diagnóstico de carcinoma de mama en cinco subtipos: carcinomas de mama de tipo basal, de tipo HER2, de tipo luminal A, de tipo luminal B y normal.

Resultados. Los carcinomas de mama más frecuentes fueron los de tipo luminal A $(62,5 \%)$, carcinomas de tipo luminal B (18\%), carcinomas de tipo HER2 (9,9\%), carcinomas de tipo basal $(8,4 \%)$ y los de fenotipo normal $(1,4 \%)$. Los carcinomas de mama de tipo luminal mostraron ser, con mayor frecuencia, de forma significativa, tumores bien diferenciados, de pequeño tamaño tumoral, con ganglios axilares negativos, estadio precoz en el momento del diagnóstico, niveles altos de BCL-2 y bajo índice de proliferación con Ki-67. En cambio, los carcinomas de mama de tipo basal y HER2 presentaban tumores de mayor tamaño, pobremente diferenciados, mayor compromiso ganglionar y estadios más avanzados en el momento del diagnóstico. Expresaban con mayor frecuencia índices de proliferación altos con Ki 67 y fueron los subtipos que en curvas de supervivencia global y de supervivencia libre de progresión mostraron un peor pronóstico.

Conclusión. La clasificación del cáncer de mama basada en parámetros inmunohistoquímicos (IHQ) permite una mejor definición pronóstica. Tanto los carcinomas de mama de tipo basal como HER2 presentan características histopatológicas e IHQ más desfavorables así como peor supervivencia y menor tiempo de recaída mientras que los carcinomas de mama de tipo luminal manifiestan características más benignas y mejor pronóstico.

Palabras clave. Cáncer de mama. Subtipos moleculares. Inmunohistoquímica.

\begin{abstract}
Background. Breast carcinomas are a heterogeneous group of tumours, in both their clinical behavior and their prognosis. The aim of this article is to classify breast carcinomas according to molecular subtypes by means of immunohistochemical markers and to analyse the clinicopathological and immunohistochemical characteristics and the patterns of survival and relapse of the different subtypes.
\end{abstract}

Methods. Two hundred and seventy-two patients diagnosed with breast cancer were classified into five subtypes: breast carcinomas of the basal type, HER2 type, luminal A type, luminal B type and normal.

Results. The most frequent breast carcinomas were: luminal A type carcinomas (62.5\%), luminal B type carcinomas (18\%), HER2 type carcinomas (9.9\%), basal type carcinomas (8.4\%) and normal phenotype carcinomas (1.4\%). Significantly and with greater frequency, the luminal type breast carcinomas proved to be well differentiated tumours, of small tumoral size, with negative axillary lymph nodes, at an early stage at the time of diagnosis, with high levels of BCL2 and a low Ki-67 proliferation index. On the contrary, the basal type and HER2 carcinomas presented larger tumours, poorly differentiated, greater ganglionar involvement and more advanced stages at the time of diagnosis. They expressed high Ki-67 proliferation indexes with greater frequency and were the subtypes that showed a worse prognosis on global survival and progression-free survival curves.

Conclusion. Breast cancer classification based on immunohistochemical (IHC) parameters makes a better prognostic definition possible. Both the basal type and the HER2 type breast carcinomas present more unfavourable histopathological and IHC characteristics, as well as a worse survival and less relapse time, while the luminal type breast carcinomas show more benign characteristics and a better prognosis.

Key words. Breast cancer. Molecular subtypes. Immunohistochemistry.
1. Centro de Salud de Irurita. Irurita (Navarra).

2. Departamento de Cirugía General. Complejo Hospitalario de Navarra. Pamplona.

3. Servicio de Anatomía Patológica. Complejo Hospitalario de Navarra. Pamplona.

4. Unidad de Metodología. Centro de Investigación Biomédica. Pamplona.

5. Departamento de Ciencias de la Salud. Universidad Pública de Navarra. Pamplona.

\section{Correspondencia}

$\mathrm{M}^{\mathrm{a}} \mathrm{a}$ Asunción Arrechea Irigoyen

Centro de Salud de Irurita

31730 Irurita (Navarra). España

e-mail: ma.arrechea.irigoyen@cfnavarra.es

Recepción: 20 de febrero de 2011

Aceptación provisional: 6 de abril de 2011

Aceptación definitiva: 7 de mayo de 2011 


\section{INTRODUCCIÓN}

El carcinoma de mama representa un grupo de tumores que muestra un comportamiento biológico muy diverso y una gran variabilidad clínica. La clasificación histológica actual de los carcinomas de mama no refleja la heterogeneidad de los tumores en su comportamiento biológico ni permite identificar los pacientes que presentarán mejores respuestas y beneficios con las diferentes modalidades terapéuticas. Actualmente se asume que, la diversidad clínica y pronóstica de carcinomas de mama que son semejantes y homogéneos en cuanto a sus factores pronósticos clásicos, se establece a nivel molecular, al expresar distintos genes que les confieren variabilidad biológica y pronóstica ${ }^{1}$. Durante los últimos años, el estudio de estos genes ha hecho posible, por un lado, comprender el comportamiento biológico del cáncer de mama y, por otro lado, individualizar el pronóstico y el tratamiento de algunos pacientes ${ }^{1,2}$.

El avance de las tecnologías de análisis genómico ha permitido clasificar los carcinomas de mama en cinco subtipos: luminal A y B, HER2-positivo, basal y similar a la mama normal ${ }^{1,3}$. Los carcinomas de mama de tipo luminal son los subtipos con mejor pronóstico y se caracterizan por expresar el gen del receptor estrogénico, genes asociados (LIV1 y ciclina D1) y queratinas de bajo peso molecular (CK7, CK8, CK18, etc.), de forma semejante al epitelio luminal de los conductos mamarios. Al expresar receptores de estrógenos (RE), estos tumores pueden tratarse con tamoxifeno o inhibidores de la aromatasa pero muestran una baja respuesta a la quimioterapia neoadyuvante. El carcinoma de mama HER2-positivo muestra expresión aumentada de genes asociados a c-erbB-2 y suele asociarse a otros marcadores de mal pronóstico, incluyendo alteraciones de otros genes como toposiomerasa II alfa, GATA4, genes de angiogénesis y proteolisis. Aunque muestran una mejor respuesta a la quimioterapia y cerca de $50 \%$ responde al tratamiento con trastuzumab, el pronóstico es malo. El subtipo basal se caracteriza por la sobreexpresión de citoqueratinas características de la capa basal (CK5/6, CK17) y la expresión de genes relacionados con la proliferación celular. Estos tumores suelen presentar mutaciones en el gen oncosupresor p53, sobreexpresan el receptor del factor de crecimiento epidérmico (EGFR) y se caracterizan por la ausencia de expresión de RE y de genes relacionados y de HER2. Este subtipo se asocia a la mutación BRCA1 y presenta el comportamiento más agresivo a pesar de su alta sensibilidad a la quimioterapia. El carcinoma de mama de tipo normal comparte características del tejido mamario normal, muestra una fuerte expresión de genes normalmente expresados en el tejido adiposo y baja expresión de genes epiteliales luminales.

Aunque en el momento actual se considera que el análisis de los perfiles de expresión génica constituye la mejor forma de clasificar los carcinomas de mama, en la mayoría de los hospitales su uso se encuentra limitado ya que son técnicas caras y difíciles de aplicar en material parafinado. En la práctica, la mayor parte de los diagnósticos de rutina se realiza mediante $\mathrm{HE}$ y técnicas de inmunohistoquímica (IHQ). Diversos estudios sugieren que, con un limitado número de marcadores inmunohistoquímicos (RE, RP, c-erbB-2, queratinas basales o de alto peso molecular, queratinas luminales o de bajo peso molecular, P63 y EGFR como marcadores más relevantes $)^{4-6}$, se pueden catalogar los carcinomas de mama en subtipos equivalentes a aquéllos basados en perfiles de expresión génica. La ventaja del estudio IHQ es que utiliza marcadores que se encuentran disponibles en la mayoría de los servicios de Anatomía Patológica y puede aplicarse sobre material archivado del que se puede obtener información clínica y evolutiva.

El objetivo principal de este trabajo es clasificar los carcinomas de mama en subtipos moleculares mediante marcadores inmunohistoquímicos y analizar las características clínicopatológicas e IHQ y los patrones de supervivencia y recaída de los distintos subtipos. 


\section{MATERIAL Y MÉTODOS}

Se incluyeron en el estudio los pacientes diagnosticados de cáncer de mama, intervenidos quirúrgicamente por el Servicio de Cirugía General del Hospital de Navarra (actual Complejo Hospitalario de Navarra) entre enero de 1998 y diciembre 1999 y enero de 2006 y julio de 2007. En los 272 casos se realizaron manualmente tissue microarrays (TMAs). Para la obtención de dichos TMAs se revisaron las tinciones de hematoxilina-eosina (HE) que contenían tejido tumoral, seleccionando la zona tumoral representativa correspondiente en el bloque de parafina. La técnica consistió en tomar un pequeño cilindro de cada bloque de parafina (bloque donante) e insertarlo de forma precisa en un bloque receptor de parafina. Mediante un sistema manual de realización de TMAs (TSK SurecutTM, Japan) se tomaron muestras de 1,6 mm de diámetro de las zonas seleccionadas más representativas del tumor en los cortes de HE. Se anotó la posición de cada cilindro, para poder identificarlo posteriormente. Se realizaron secciones de $4 \mu \mathrm{m}$ y se tiñeron con HE para comprobar la presencia de tejido tumoral. En los cortes de $4 \mu \mathrm{m}$ de parafina de los TMAs se realizaron estudios de IHQ.

Dado que en el momento actual no existe consenso sobre el panel IHQ más adecuado para definir los subtipos moleculares, optamos por una clasificación aceptada por la mayoría de autores basada, tanto en la expresión de RE, receptor de progesterona (RP) y HER2, como en la expresión de proteínas características de las células basales/mioepiteliales (CK5/6, CK14, p63 y EGFR). De acuerdo con el estado de estos marcadores se definieron cinco subtipos: luminal A (RE+, RP+/-, HER2-), luminal B (RE+, RP+/-, HER2+), HER2 (RE-, RP-, HER2 +), basal (RE-, RP-, HER2- y CK5/6, CK14, p63 y/o EGFR+) y normal (RE-, RP-, HER2-, CK5/6-, CK14-, p63- y/o EGFR-). En todos los casos se determinaron variables tanto clinicopatológicas (edad, sexo, tamaño tumoral, tipo histológico, grado, estado ganglionar axilar, tipo de cirugía realizada, localización y cuadrante afectado, ganglio centinela, estadio tumoral y tratamiento adyuvante) como IHQ (además de los anteriormente mencionados, se estudió la expresión de BCL-2, p53, Ki-67, MUC1 y COX-2, en base a estudios que indican la relación de dichos marcadores con el pronóstico del cáncer de mama).

El diagnóstico histopatológico definitivo lo realizaron dos patólogos del Hospital de Navarra. La valoración IHQ de RE, RP, ecadherina, EGFR, CK5/6 y CK14 se consideró positiva cuando la expresión superaba el $10 \%$ de las células tumorales; la valoración IHQ de BCL2, p53, p63, COX2 y MUC1 cuando su expresión superaba el $5 \%$ de las células. En el caso de HER2, la valoración fue semicuantitativa; se consideró positivo el caso de $3+$ y negativo el de 0 o $1+$. En los casos de 2+ se realizó valoración mediante técnica FISH para determinar si era positivo o negativo. Para la interpretación de ki-67 se establecieron 3 categorías: índice de proliferación bajo (menor del 15\% de las células positivas), índice de proliferación medio (entre 15-25\% de las células positivas) e índice de proliferación alto (más del 25\% de las células positivas). Las características de todos los marcadores IHQ estudiados en tejido tumoral, clones de anticuerpos, sistemas de recuperación antigénica y diluciones se resumen en la tabla 1.

Aunque se considera que un periodo de tiempo de 5 años puede ser suficiente para obtener resultados al analizar las tasas de recaída, se estima que el tiempo medio de un estudio de supervivencia en cáncer de mama debe ser en torno a los 10 años de seguimiento. Por ello, el análisis de las curvas de supervivencia libre de progresión y de supervivencia global se realizó en los 125 casos estudiados en los años 1998-1999, con un seguimiento máximo de 11 años y medio y un tiempo medio de 10 años y medio.

La descripción de las variables clinicopatológicas e IHQ de la muestra se realizó mediante frecuencias y porcentajes. La asociación de estas variables con fenotipo molecular se analizó mediante el test $\mathrm{X}^{2}$, el test de Fisher, según correspondiera, y se 
complementó mediante el ajuste de modelos de regresión logística binaria y logística ordinal, considerando como variable explicativa el fenotipo molecular, que permitió derivar odds-ratios y sus intervalos de confianza al $95 \%$. El estudio de recaída y supervivencia durante el seguimiento se realizó mediante curvas de Kaplan-Meier, que fueron comparadas usando el test de log-rank, y se complementó con el ajuste de modelos de regresión de Cox, que permitieron la estimación de las razones de riesgo (hazard ratios) con sus intervalos de confianza al 95\%. Los análisis estadísticos se realizaron mediante el programa $R$, versión 2.5.1.

Tabla 1. Características de los marcadores inmunohistoquímicos

\begin{tabular}{|c|c|c|c|c|}
\hline Anticuerpo & Clon & Dilución & $\begin{array}{l}\text { Recuperación } \\
\text { antigénica }\end{array}$ & Laboratorio \\
\hline $\mathrm{RE}$ & $6 \mathrm{~F} 11$ & pre diluido & $4 \mathrm{~min}$ & ATOM \\
\hline $\mathrm{RP}$ & $1^{\mathrm{a}} 6$ & pre diluido & $4 \mathrm{~min}$ & ATOM \\
\hline c-erbB-2 & CB11 & $1: 50$ & $4 \mathrm{~min}$ & ATOM \\
\hline E-cadherina & $36 \mathrm{~B} 5$ & pre diluido & $3 \mathrm{~min}$ & Menarini \\
\hline BCL2 & BCL2/100/D5 & $1: 80$ & $60 \mathrm{~min} . \mathrm{T}^{\mathrm{a}} 25^{\circ} \mathrm{C}$ & Novocastra \\
\hline p-53 & BP53-11 & pre diluido & $4 \min$ & ATOM \\
\hline Ki-67 & MIB-1 & $1: 50$ & $4 \min$ & Master Diagnóstica \\
\hline EGFR & $2-18 \mathrm{C} 9$ & pre diluido & Proteasa K & DAKO \\
\hline $\mathrm{CK} 5 / 6$ & D5/16B4 & pre diluido & $4 \mathrm{~min}$ & Master Diagnóstica \\
\hline CK14 & $34 \mathrm{BE} 12$ & $1: 50$ & $3 \min$ & DAKO \\
\hline p63 & JUL & $1: 25$ & $4 \min$ & Master Diagnóstica \\
\hline COX-2 & SP21 & $1: 100$ & $32 \min \mathrm{T}^{\mathrm{a}} \mathrm{amb}$ & Diagnostic Biosystems \\
\hline MUC1 & Ma695 & $1: 100$ & $60 \min . \mathrm{T}^{\mathrm{a}} 25^{\mathrm{a}} \mathrm{C}$ & Novocatra \\
\hline
\end{tabular}

\section{RESULTADOS}

Se estudiaron 272 carcinomas de mama que fueron reclasificados según la clasificación de Perou y $\operatorname{col}^{1}$. En la tabla 2 se detallan las características clínicopatológicas según el fenotipo molecular. Los carcinomas más frecuentes fueron los carcinomas de mama de tipo luminal A (62,5\%), seguidos de los carcinomas de tipo luminal B (18\%), los carcinomas de tipo HER2 (9,9\%), los carcino- mas de tipo basal $(8,4 \%)$ y los de fenotipo normal (1,4\%). La edad media de presentación del cáncer de mama en nuestro estudio fue de 59,8 años (rango de 29-94 años), aunque no se observaron diferencias estadísticamente significativas en cuanto a la edad en los cinco subtipos moleculares.

El análisis en esta serie mostró una asociación estadísticamente significativa entre los fenotipos del carcinoma de mama y el tamaño tumoral $(\mathrm{p}=0,004)$ observándose 
que, en el momento del diagnóstico, los carcinomas de mama de tipo luminal presentan un tamaño tumoral más pequeño que los de tipo basal y los de tipo HER2. El $67,6 \%$ y el $59,1 \%$ de los carcinomas de mama de tipo luminal A y $\mathrm{B}$ respectivamente tenían un tamaño inferior a $2 \mathrm{~cm}$ mientras que únicamente el $22,7 \%$ de los carcinomas de mama de tipo basal tenía menos de $2 \mathrm{~cm}$ (pT1). El 77,2\% de los carcinomas de mama de tipo basal presentaba un tamaño superior a $2 \mathrm{~cm}$. En los carcinomas de mama de tipo HER2, los tumores mayores de $2 \mathrm{~cm}$ representaron el $52 \%$ mientras que los menores de $2 \mathrm{~cm}$, el $48 \%$.

$\mathrm{Al}$ categorizar los carcinomas de mama según el subtipo histológico las diferencias encontradas en los diferentes subgrupos moleculares no fueron estadísticamente significativas $(p=0,16)$. En todos los subtipos moleculares, el carcinoma ductal infiltrante (CDI) fue el subtipo histológico predominante. Los carcinomas de tipo lobulillar infiltrante (CLI) se observaron sobre todo en los subtipos luminales $(14,7 \%$ de casos en el subtipo luminal A, 8,1\% de casos en el subtipo luminal B); en los carcinomas de mama de tipo basal no se observaron CLI mientras que en los carcinomas de tipo HER2 sólo un 3,7\% de los casos era CLI.

El grado histológico se asoció de forma significativa al subtipo molecular $(\mathrm{p}=0,0004)$. Los carcinomas de mama bien diferenciados se observaron con mayor frecuencia en los carcinomas de tipo luminal A. El 43\% de los carcinomas de mama de tipo luminal A era grado I, el $41 \%$ grado II y únicamente el 16\% era grado III. Los carcinomas de mama de tipo basal y los de tipo HER2 presentaban con mayor frecuencia tumores peor diferenciados. El 70\% de los carcinomas de mama subtipo basal $(n=14)$ era grado III y el $25 \%$ grado II. En cuanto a los carcinomas de mama tipo HER2, el $66,6 \%$ era de grado III.

El tipo de intervención quirúrgica viene determinada por el tipo, tamaño del tumor y focalidad tumoral. En lo que se refiere al tipo de cirugía, se observaron diferencias estadísticamente significativas en los sub- tipos moleculares $(\mathrm{p}=0,0014)$. Se realizó un mayor porcentaje de intervenciones conservadoras en los subtipos luminal A y B (65\% y $63 \%$ respectivamente) mientras que en los subtipos basal y HER2 se realizó mayor proporción de cirugía radical (72,7\% y $55,5 \%$ respectivamente).

No se observó asociación estadísticamente significativa entre los fenotipos tumorales y el cuadrante afecto, la localización tumoral y la afectación del ganglio centinela. En cambio las diferencias en los distintos subtipos moleculares fueron estadísticamente significativas en cuanto al compromiso de ganglios axilares. El subtipo basal y el subtipo HER2 presentaron un compromiso ganglionar significativamente mayor que el resto de subtipos $(\mathrm{p}=0,004)$. La afectación ganglionar en el momento del diagnóstico en los carcinomas de mama de tipo luminal A fue del 27,6\%; en los carcinomas de mama de tipo luminal B del $42,8 \%$; en los carcinomas de mama de tipo HER2 del $51,8 \%$ y en los de tipo basal del $54,5 \%$.

En los distintos subtipos, se encontraron diferencias estadísticamente significativas $(\mathrm{p}=0,0009)$ en cuanto al estadio tumoral en el momento del diagnóstico. La mayor parte de los carcinomas de mama de tipo luminal A $(54,1 \%)$ se encontraba en estadio tumoral I mientras que, tan sólo el $18,2 \%$ y el $26 \%$ de los carcinomas de mama subtipo basal y HER2 respectivamente, se encontraban en estadio I. El 50\% de los carcinomas de mama de tipo basal fue catalogado en estadio II y el $31,8 \%$ en estadio III.

En la tabla 3 se resumen los resultados del análisis del grado de asociación entre los subtipos y los marcadores IHQ. Dado que los parámetros RE, RP y c-erbB-2 se han utilizado para la definición de fenotipo no procede modelizar estos datos ya que la asociación es perfecta o casi perfecta. La expresión de BCL2 se asocia de forma significativa a los carcinomas de mama de fenotipo luminal A y luminal B. En cambio, los carcinomas de mama de tipo basal se asocian con mayor frecuencia a la expresión de EGFR, CK14, CK5/6 y valores altos de Ki-67. 
Tabla 2. Asociación de los parámetros clínico-patológicos con el fenotipo molecular del cáncer de mama

\begin{tabular}{|c|c|c|c|c|c|c|}
\hline Parámetro & $\begin{array}{c}\text { Basal } \\
\mathrm{N}=22(8,4 \%)\end{array}$ & $\begin{array}{c}\text { HER2 } \\
\mathrm{N}=27(9,9 \%)\end{array}$ & $\begin{array}{c}\text { Luminal A } \\
\mathrm{N}=170(62,5 \%)\end{array}$ & $\begin{array}{c}\text { Luminal B } \\
\mathrm{N}=49(18 \%)\end{array}$ & $\begin{array}{c}\text { Normal } \\
\mathrm{N}=4(1,4 \%)\end{array}$ & p-valor \\
\hline Edad & & & & & & 0,378 \\
\hline$\leq 50$ años & $3(13,6 \%)$ & $8(30 \%)$ & $44(25,8 \%)$ & $16(32,6 \%)$ & $0(0 \%)$ & \\
\hline$>50$ años & $19(86,3 \%)$ & $19(70 \%)$ & $126(74,2 \%)$ & $33(67,3 \%)$ & $4(100 \%)$ & \\
\hline Tamaño tumoral & & & & & & 0,004 \\
\hline pT1 & $5(22,7 \%)$ & $13(48,1 \%)$ & $115(67,6 \%)$ & $29(59,1 \%)$ & $1(25 \%)$ & \\
\hline pT2 & $13(59 \%)$ & $8(29,6 \%)$ & $44(25,8 \%)$ & $18(36,7 \%)$ & $3(75 \%)$ & \\
\hline pT3 & $3(13,6 \%)$ & $3(11,1 \%)$ & $5(3 \%)$ & $0(0 \%)$ & $0(0 \%)$ & \\
\hline pT4 & $1(4,5 \%)$ & $3(11,1 \%)$ & $6(3,5 \%)$ & $2(4,1 \%)$ & $0(0 \%)$ & \\
\hline Tipo histológico & & & & & & 0,166 \\
\hline CLI & $0(0 \%)$ & $1(4 \%)$ & $25(14 \%)$ & $4(8,2 \%)$ & $0(0 \%)$ & \\
\hline $\mathrm{CDI}$ & $20(91 \%)$ & $22(81 \%)$ & $108(64 \%)$ & $34(69,4 \%)$ & $4(100 \%)$ & \\
\hline Otros & $2(9 \%)$ & $4(15 \%)$ & $37(22 \%)$ & $11(22,4 \%)$ & $0(0 \%)$ & \\
\hline Grado & & & & & & 0,0004 \\
\hline I & $1(5 \%)$ & $3(12,5 \%)$ & $56(43 \%)$ & $17(38 \%)$ & $0(0 \%)$ & \\
\hline II & $5(25 \%)$ & $5(20,8 \%)$ & $53(41 \%)$ & $19(42 \%)$ & $1(25 \%)$ & \\
\hline III & $14(70 \%)$ & $16(66,6 \%)$ & $21(16 \%)$ & $9(20 \%)$ & $3(75 \%)$ & \\
\hline Tipo operación & & & & & & 0,0014 \\
\hline Radical & $16(72,7 \%)$ & $15(55,5 \%)$ & $59(35 \%)$ & $18(37 \%)$ & $3(75 \%)$ & \\
\hline Conservadora & $6(27,2 \%)$ & $12(44,4 \%)$ & $111(65 \%)$ & $31(63 \%)$ & $1(25 \%)$ & \\
\hline Cuadrante & & & & & & 0,38 \\
\hline CSE & $10(48 \%)$ & $7(26 \%)$ & $57(33,5 \%)$ & $13(27 \%)$ & $1(25 \%)$ & \\
\hline CSI & $1(4,5 \%)$ & $1(3,7 \%)$ & $13(7,6 \%)$ & $9(18,3 \%)$ & $0(0 \%)$ & \\
\hline CIE & $1(4,5 \%)$ & $3(11,1 \%)$ & $13(7,6 \%)$ & $2(4 \%)$ & $0(0 \%)$ & \\
\hline CII & $1(4,5 \%)$ & $3(11,1 \%)$ & $6(3,5 \%)$ & $7(14,3 \%)$ & $0(0 \%)$ & \\
\hline ICS & $4(18,2 \%)$ & $3(11,1 \%)$ & $27(15,9 \%)$ & $4(8,1 \%)$ & $1(25 \%)$ & \\
\hline ICE & $3(13,6 \%)$ & $5(18,5 \%)$ & $17(10 \%)$ & $7(14,3 \%)$ & $1(25 \%)$ & \\
\hline ICInt & $0(0 \%)$ & $1(3,7 \%)$ & $11(6,4 \%)$ & $1(2 \%)$ & $0(0 \%)$ & \\
\hline ICInf & $0(0 \%)$ & $2(7,4 \%)$ & $7(4,1 \%)$ & $4(8,1 \%)$ & $0(0 \%)$ & \\
\hline Otros & $2(9,1 \%)$ & $2(7,4 \%)$ & $19(11,1 \%)$ & $2(4 \%)$ & $1(25 \%)$ & \\
\hline
\end{tabular}




\begin{tabular}{|c|c|c|c|c|c|c|}
\hline Parámetro & $\begin{array}{c}\text { Basal } \\
\mathrm{N}=22(8,4 \%)\end{array}$ & $\begin{array}{c}\text { HER2 } \\
\mathrm{N}=27(9,9 \%)\end{array}$ & $\begin{array}{c}\text { Luminal A } \\
\mathrm{N}=170(62,5 \%)\end{array}$ & $\begin{array}{c}\text { Luminal B } \\
\mathrm{N}=49(18 \%)\end{array}$ & $\begin{array}{c}\text { Normal } \\
\mathrm{N}=4(1,4 \%)\end{array}$ & p-valor \\
\hline Localización & & & & & & 0,43 \\
\hline MD & $9(41 \%)$ & $17(63 \%)$ & $77(45,3 \%)$ & $23(47 \%)$ & $1(25 \%)$ & \\
\hline MI & $13(59 \%)$ & $10(37 \%)$ & $91(53,5 \%)$ & $26(53 \%)$ & $3(75 \%)$ & \\
\hline Ganglio centinela & & & & & & 0,28 \\
\hline- & $5(22,7 \%)$ & $3(11,1 \%)$ & $43(25,3 \%)$ & $9(18,3 \%)$ & $0(0 \%)$ & \\
\hline+ & $4(18,2 \%)$ & $2(7,4 \%)$ & $17(10 \%)$ & $9(18,3 \%)$ & $0(0 \%)$ & \\
\hline $\mathrm{NH}$ & $13(59 \%)$ & $22(81,5 \%)$ & $110(65 \%)$ & $31(63,2 \%)$ & $4(100 \%)$ & \\
\hline Ganglios afectos & & & & & & 0,004 \\
\hline $\mathrm{pNx}$ & $0(0 \%)$ & $3(11,1 \%)$ & $29(17 \%)$ & $6(12,2 \%)$ & $0(0 \%)$ & \\
\hline pNO & $10(45,4 \%)$ & $10(37 \%)$ & $95(56 \%)$ & $22(44,9 \%)$ & $1(25 \%)$ & \\
\hline $\mathrm{pN} 1$ & $5(22,7 \%)$ & $11(40,7 \%)$ & $31(18,2 \%)$ & $13(26,5 \%)$ & $0(0 \%)$ & \\
\hline $\mathrm{pN} 2$ & $4(18,2 \%$ & $2(7,4 \%)$ & $11(6,4 \%)$ & $5(10,2 \%)$ & $1(25 \%)$ & \\
\hline $\mathrm{pN} 3$ & $3(13,6 \%)$ & $1(3,7 \%)$ & $4(3 \%)$ & $3(6,1 \%)$ & $2(50 \%)$ & \\
\hline Estadio & & & & & & 0,0009 \\
\hline I & $4(18,2 \%)$ & 7 (26\%) & $92(54,1 \%)$ & $20(40,8 \%)$ & $0(0 \%)$ & \\
\hline II & $11(50 \%)$ & $14(51,8 \%)$ & $59(34,7 \%)$ & $20(40,8 \%)$ & $1(25 \%)$ & \\
\hline III & $7(31,8 \%)$ & $6(22,2 \%)$ & $19(11,2 \%)$ & $9(18,4 \%)$ & $3(75 \%)$ & \\
\hline Quimioterapia & & & & & & 0,0019 \\
\hline No & $4(18 \%)$ & $4(15 \%)$ & $80(47 \%)$ & $17(35 \%)$ & $2(50 \%)$ & \\
\hline Sí & $18(82 \%)$ & $23(85 \%)$ & $89(53 \%)$ & $32(65 \%)$ & $2(50 \%)$ & \\
\hline Radioterapia & & & & & & 0,0019 \\
\hline No & $9(41 \%)$ & $11(41 \%)$ & $48(28 \%)$ & $13(27 \%)$ & $2(50 \%)$ & \\
\hline Sí & $13(59 \%)$ & $16(59 \%)$ & $121(72 \%)$ & $36(73 \%)$ & $2(50 \%)$ & \\
\hline
\end{tabular}

pT1: tumor de 2,0 cm ó menos de diámetro máximo, pT2: tumor de más de 2,0 cm y menos de 5,0 cm, pT3: tumor de más de 5,0 cm de diámetro máximo, pT4: tumor de cualquier tamaño con extensión a pared torácica o a piel, CLI: carcinoma lobulillar infiltrante, CDI: carcinoma ductal infiltrante, CSE: cuadrante supero-externo, CSI: cuadrante superointerno, CIE: cuadrante ínfero-externo, CII: cuadrante ínfero-interno, ICS: intercuadrante superior, ICE: intercuadrante externo, ICInt: intercuadrante interno, ICInf: intercuadrante inferior, pNx: No se puede valorar la afectación ganglionar, pN0: no hay metástasis ganglionares regionales en el estudio histológico, pN1: metástasis en 1 a 3 ganglios linfáticos axilares y/o en ganglios de la mamaria interna, pN2: metástasis en 4 a 9 ganglios linfáticos, pN3: metástasis en 10 ó más ganglios linfáticos. 
Tabla 3. Asociación de los parámetros inmunohistoquímicos con el fenotipo molecular (regresión logística) (OD: odss ratio, IC: intervalo de confianza)

\begin{tabular}{|c|c|c|c|c|}
\hline Parámetro & Estimación & OR & IC: $95 \%$ & p-valor \\
\hline BCL2 & & & & $\mathrm{L}=82,521, \mathrm{p}<0,001$ \\
\hline Basal* $^{*}$ & - & 1 & - & \\
\hline HER2 & $-0,501$ & 0,60 & $(0,157-2,338)$ & 0,467 \\
\hline Luminal A & 3,114 & 22,51 & $(7,819-64,856)$ & $<0,001$ \\
\hline Luminal B & 2,107 & 8,22 & $(2,624-25,762)$ & $<0,001$ \\
\hline Normal & 2,079 & 8,00 & $(0,690-92,702)$ & 0,096 \\
\hline p53 & & & & $\mathrm{L}=3,809, \mathrm{p}=0,433$ \\
\hline Basal & - & 1 & - & \\
\hline HER2 & $-0,288$ & 0,75 & $(0,219-2,574)$ & 0,647 \\
\hline Luminal A & $-0,501$ & 0,60 & $(0,226-1,627)$ & 0,320 \\
\hline Luminal B & $-0,609$ & 0,54 & $(0,181-1,630)$ & 0,277 \\
\hline Normal & $-2,079$ & 0,12 & $(0,011-1,448)$ & 0,096 \\
\hline EGFR & & & & $\mathrm{L}=39,741, \mathrm{p}<0,001$ \\
\hline Basal & - & 1 & - & \\
\hline HER2 & $-1,499$ & 0,22 & $(0,0614-0,812)$ & 0,022 \\
\hline Luminal A & $-2,853$ & 0,057 & $(0,019-0,172)$ & $<0,001$ \\
\hline Luminal B & $-2,881$ & 0,056 & $(0,015-0,203)$ & $<0,001$ \\
\hline Normal & $-17,729$ & $<0,001$ & (0-inf) & 0,98 \\
\hline p63 & & & & $\mathrm{L}=1,643, \mathrm{p}=0,801$ \\
\hline Basal & - & 1 & - & \\
\hline HER2 & 0,642 & 1,90 & $(0,311-11,607)$ & 0,487 \\
\hline Luminal A & 0,229 & 1,25 & $(0,270-5,852)$ & 0,770 \\
\hline Luminal B & 0,147 & 1,15 & $(0,206-6,520)$ & 0,867 \\
\hline Normal & $-14,315$ & $<0,001$ & (0-inf) & 0,990 \\
\hline CK14 & & & & $\mathrm{L}=10,865, \mathrm{p}=0,028$ \\
\hline Basal & - & 1 & - & \\
\hline HER2 & $-0,194$ & 0,82 & $(0,233-2,915)$ & 0,763 \\
\hline Luminal A & $-1,268$ & 0,28 & $(0,101-0,786)$ & 0,016 \\
\hline Luminal B & $-0,464$ & 0,63 & $(0,203-1,950)$ & 0,422 \\
\hline Normal & $-15,873$ & $<0,001$ & (0-inf) & 0,989 \\
\hline CK5/6 & & & & $\mathrm{L}=27,449, \mathrm{p}<0,001$ \\
\hline Basal & - & 1 & - & \\
\hline HER2 & 0,049 & 1,05 & $(0,321-3,436)$ & 0,936 \\
\hline Luminal A & $-1,749$ & 0,17 & $(0,067-0,452)$ & 0,001 \\
\hline Luminal B & $-1,014$ & 0,36 & $(0,126-1,049)$ & 0,061 \\
\hline Normal & $-15,854$ & $<0,001$ & (0-inf) & 0,983 \\
\hline
\end{tabular}




\begin{tabular}{c|ccc|c}
\hline Parámetro & Estimación & OR & IC: 95\% & p-valor \\
\hline COX-2 & & & & L=3,995, $\mathrm{p}=0,407$ \\
Basal & - & 1 & $(0,724-8,635)$ & \\
\hline HER2 & 0,916 & 2,50 & $(0,389-2,917)$ & 0,147 \\
Luminal A & 0,063 & 1,065 & $(0,433-4,105)$ & 0,903 \\
Luminal B & 0,288 & 1,33 & $(0,0717-9,688)$ & 0,616 \\
Normal & $-0,182$ & 0,83 & & 0,884 \\
\hline MUC1 & & & - & $\mathrm{L}=0,687, \mathrm{p}=0,953$ \\
Basal & - & 1 & $(0,067-19,601)$ & 0,923 \\
\hline HER2 & 0,140 & 1,15 & $(0,097-6,699)$ & 0,841 \\
Luminal A & $-0,216$ & 0,80 & $(0,094-12,849)$ & 0,939 \\
Luminal B & 0,095 & 1,1 & $(0-i n f)$ & 0,991 \\
Normal & 13,570 & $7,82 \times 105$ & & $\mathrm{~L}=25,203 \mathrm{p}<0,001$ \\
\hline Ki-67 & & & - & \\
Basal & - & 1 & $(0,209-1,944)$ & 0,433 \\
\hline HER2 & $-0,445$ & 0,64 & $(0,067-0,414)$ & $<0,001$ \\
Luminal A & $-1,782$ & 0,17 & $(0,113-6,760)$ & 0,001 \\
Luminal B & $-1,719$ & 0,18 & & 0,897 \\
Normal & $-0,133$ & 0,87 &
\end{tabular}

* Se ha tomado el fenotipo basal como categoría de referencia.

Los CDI y los CLI constituían el $80,1 \%$ del total de carcinomas de mama. Por ello, para la valoración estadística establecimos tres grupos: CDI $(69,1 \%)$, CLI (11\%) y el resto de carcinomas de mama (en los que se incluyeron el carcinoma ductal in situ, carcinoma mucinoso carcinoma tubular, carcinoma papilar, carcinoma mixto ductal-lobulillar, carcinoma medular, carcinoma inflamatorio, cistosarcoma filodes y carcinoma rico en glucógeno) (19,9\%). $\mathrm{Al}$ analizar las diferencias pronósticas en función de estos tres grandes subgrupos histológicos no se observaron diferencias estadísticamente significativas en cuanto al riesgo de recaídas $(\mathrm{p}=0,161)$ ni de supervivencia ( $p=0,290)$ (Fig. 1, Tabla 4). Sin embargo, cuando utilizamos la clasificación del cáncer de mama según los criterios de Perou y col, los resultados revelan diferencias estadísticamente significativas en el pronóstico de los distintos subtipos moleculares de cáncer de mama. Al analizar las curvas de supervivencia global (SG) y la su- pervivencia libre de progresión (SLP), los carcinomas de mama de tipo basal y HER2 tienen peor pronóstico que los carcinomas de mama de tipo luminal (Fig. 2, Tabla 4). Las diferencias entre los fenotipos moleculares del cáncer de mama son claras, siendo los carcinomas de mama de tipo luminal $A$, seguido de los de tipo luminal $B$, los que mayor SLP y mayor SG presentan, con un riesgo de recaída para los carcinomas de mama de tipo luminal A de 0,16 (6 veces menor) en comparación con los de tipo basal, y con un riesgo de mortalidad para carcinomas de mama de tipo luminal A de 0,10 (10 veces menor) en comparación con los de tipo basal. Los carcinomas de mama de tipo luminal $B$ tienen un riesgo de recaída 3 veces menor $(H R=0,34)$ y un riesgo de mortalidad 5 veces menor $(\mathrm{HR}=0,20)$ que el subtipo basal. A su vez, los carcinomas de mama de tipo HER2 tienen un riesgo de recaída ligeramente inferior al basal $(\mathrm{HR}=0,66)$ y un riesgo de mortalidad 2 veces menor $(H R=0,20)$ que el subtipo basal. 
a) SLP según diagnóstico histológico clásico

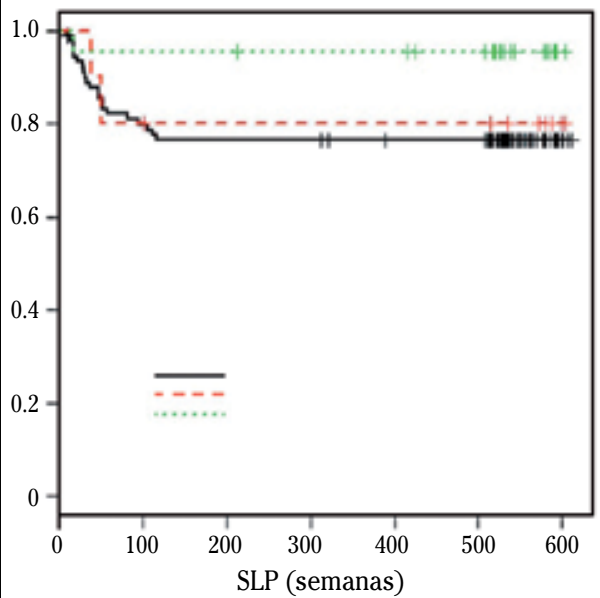

b) SG según diagnóstico histológico clásico

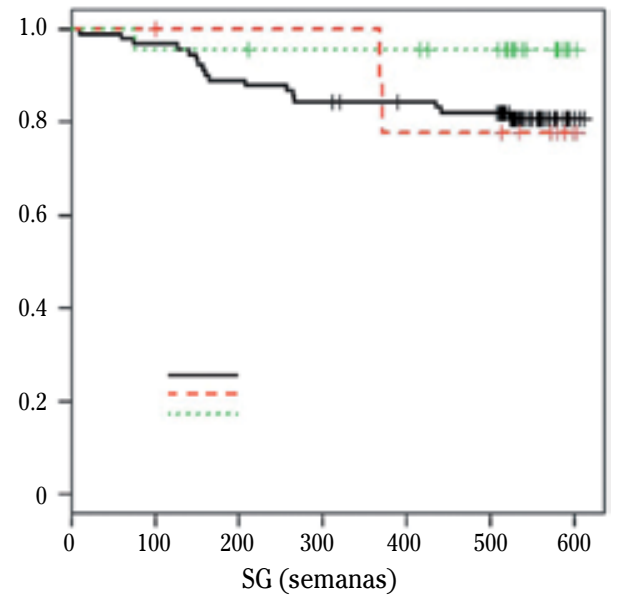

Figura 1. Curvas de Kaplan-Meier de la supervivencia libre de progresión (SLP) y supervivencia global (SG) según el diagnóstico histológico clásico (CLI: carcinoma lobulillar infiltrante, CDI: carcinoma ductal infiltrante).

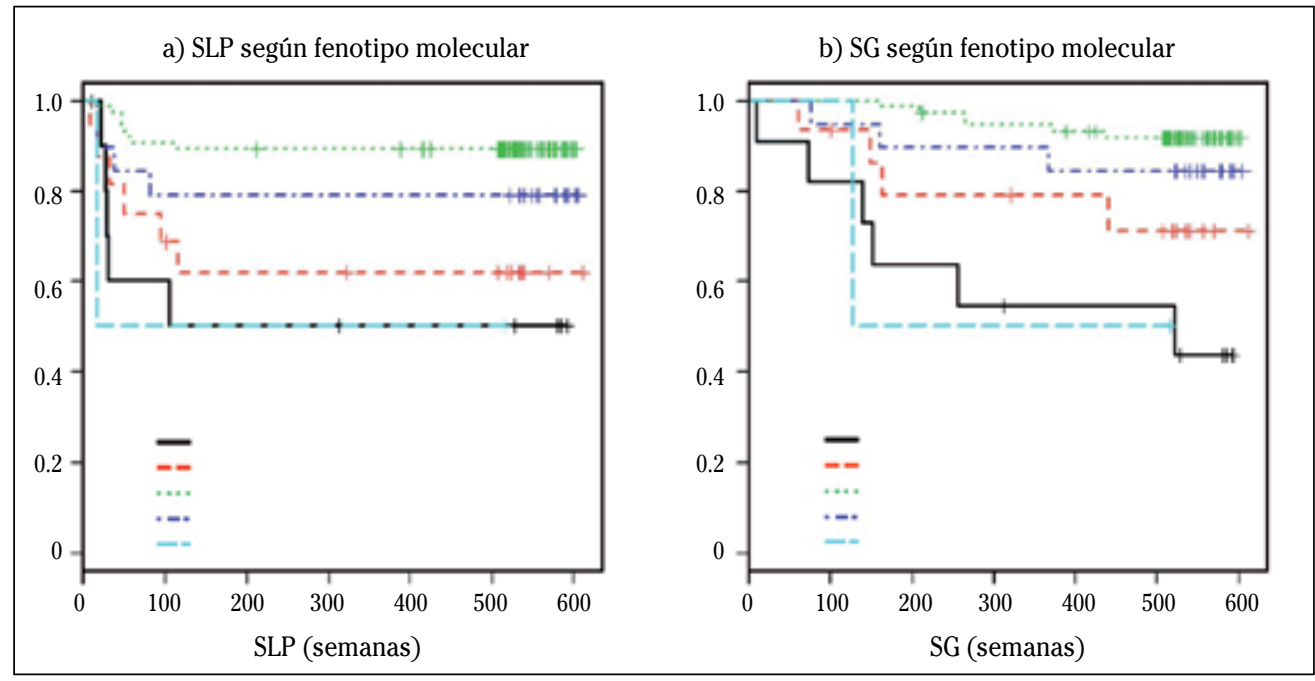

Figura 2. Curvas de Kaplan-Meier de la supervivencia libre de progresión (SLP) y supervivencia global (SG) según el fenotipo molecular.

Al comparar los carcinomas de mama del periodo 2006-2007 con los carcinomas del periodo 1998-1999 se observa una tendencia en el Hospital de Navarra a diagnosticar los carcinomas de mama en fases más precoces, con mejores perspectivas de respuesta adecuada al tratamiento. En los años más recientes se tienden a diagnosticar tumores de menor tamaño (el tamaño medio tumoral en los años 2006-2007 fue de 1,81 cm versus 2,05 cm en los años 1998-1999), con mejor diferenciación tumoral (48\% de tumores de grado I en 2006-2007 versus $18,6 \%$ en $1998-99$ y $52 \%$ de tumores grado II 
y III en 2006-2007 versus $81 \%$ en 1998-1999), con menor porcentaje de afectación ganglionar $(9,6 \%$ de carcinomas de mama con más de 4 ganglios afectos en 2006-2007 versus
$17,6 \%$ en 1998-99) y con menor porcentaje de carcinomas de mama en estadios avanzados (13\% de tumores en estadio III en 20062007 versus $20 \%$ en $1998-99$ ).

Tabla 4. Resultados de recaída y supervivencia según el fenotipo molecular o según el diagnóstico histológico (modelos de regresión de Cox)

\begin{tabular}{|c|c|c|c|c|}
\hline Parámetro & Estimación & HR & IC: $95 \%$ & p-valor \\
\hline $\begin{array}{l}\text { Recaída según fenotipo } \\
\text { Basal * }\end{array}$ & 1 & 1 & & 0,002 \\
\hline HER2 & $-0,407$ & 0,66 & $(0,203-2,186)$ & 0,502 \\
\hline Luminal A & $-1,818$ & 0,16 & $(0,052-0,498)$ & 0,001 \\
\hline Luminal B & $-1,065$ & 0,34 & $(0,092-1,285)$ & 0,112 \\
\hline Normal & 0,199 & 1,22 & $(0,142-10,45)$ & 0,856 \\
\hline $\begin{array}{l}\text { Recaída según diagnóstico } \\
\text { histológico }\end{array}$ & & & & 0,161 \\
\hline CDI & 1 & 1 & & \\
\hline CLI & $-0,183$ & 0,83 & $(0,19-3,55)$ & 0,804 \\
\hline Otros & $-1,730$ & 0,17 & $(0,02-1,318)$ & 0,091 \\
\hline $\begin{array}{l}\text { Supervivencia según } \\
\text { fenotipo }\end{array}$ & & & & $<0,001$ \\
\hline Basal & 1 & 1 & & \\
\hline HER2 & $-0,897$ & 0,41 & $(0,114-1,44)$ & 0,165 \\
\hline Luminal A & $-2,298$ & 0,10 & $(0,032-0,312)$ & $<0,001$ \\
\hline Luminal B & $-1,597$ & 0,20 & $(0,05-0,812)$ & 0,024 \\
\hline Normal & 0,035 & 1,03 & $(0,124-8,644)$ & 0,974 \\
\hline $\begin{array}{l}\text { Supervivencia según } \\
\text { diagnóstico histológico }\end{array}$ & & & & 0,290 \\
\hline CDI & 1 & 1 & & \\
\hline CLI & 0,062 & 1,06 & $(0,245-4,608)$ & 0,804 \\
\hline Otros & $-1,468$ & 0,23 & $(0,0306-1,73)$ & 0,091 \\
\hline
\end{tabular}

CLI: carcinoma lobulillar infiltrante, CDI: carcinoma ductal infiltrante, HR: hazard ratio, IC: intervalo de confianza).

* Se ha tomado el fenotipo basal como categoría de referencia.

\section{DISCUSIÓN}

En el presente estudio se observó que el fenotipo molecular predominante numéricamente fue el luminal $\mathrm{A}$. La información recogida por Blows y col$^{7}$ de 10.159 casos de carcinoma de mama procedentes de doce estudios diferentes, reveló que el 78\% del total de casos (7.243 casos) correspondía a carcinomas de mama de tipo luminal. De ellos, el 92\% era de tipo luminal A (7.882 casos) y el $8 \%$ era de tipo luminal B (639 casos). Nuestra serie muestra un porcentaje similar, el 80,5\% de los casos era de tipo luminal (219 casos), con una distribución ligeramente diferente, siendo el $77,6 \%$ de tipo luminal A (170 casos) y el $22,3 \%$ de tipo luminal B (49 casos). El 9,9\% de los pacientes de nuestro estudio (27 casos) corresponde a carcinomas de mama de tipo HER2, porcentaje inferior al observado en la literatura internacional, donde se ha mostrado que $20-30 \%$ de los carcinomas de mama expresa HER2 ${ }^{8,9}$. Sin embargo, en 
el estudio de Blows y $\mathrm{col}^{7}$ tan sólo el 6,2\% (632 casos) corresponde a carcinomas de fenotipo HER2. En nuestra serie, el grupo de carcinomas de mama de fenotipo basal constituye el $8,4 \%$ (22 casos). En la literatura médica el porcentaje de carcinomas de mama de fenotipo basal en mujeres caucásicas varía en los diferentes estudios y oscila entre el $4,2 \%$ y el $26,8 \%{ }^{10}$. La población de nuestro estudio es de forma predominante caucásica, muy diferente de la observada en el estudio de Carey y col $^{11}$ en el que la población mayoritaria es afroamericana y en donde se observa una mayor prevalencia del subtipo basal (39\% en las afroamericanas premenopáusicas, 14\% en las afroamericanas posmenopáusicas y $16 \%$ en las mujeres no afroamericanas de todas las edades).

Los resultados obtenidos en nuestro estudio están plenamente de acuerdo con lo referido en trabajos anteriores ${ }^{12}$. Un porcentaje amplio de los carcinomas de mama de tipo luminal (tanto los de tipo A como los de tipo B) mostraron de forma significativa características favorables como tamaño tumoral pequeño (de menos de 2 $\mathrm{cm}$ ), tumores diferenciados con bajo a moderado grado histológico, ganglios axilares negativos y estadio precoz en el momento del diagnóstico. Tanto el subtipo luminal A como el B expresaban niveles altos de BCL-2 y bajos índices de proliferación con $\mathrm{Ki}-67$, todo ello de manera altamente significativa. Los carcinomas de mama de tipo luminal fueron los subtipos con mejor pronóstico y dentro de los carcinomas de tipo luminal, los carcinomas de mama de tipo luminal A mostraron mayor supervivencia y menor riesgo de recaída que los que los carcinomas de mama de tipo luminal B.

Nuestro estudio coincide con otros en que los carcinomas de mama de tipo basal y los de tipo HER2 tienen peores características clinicopatológicas e inmunohistoquímicas $^{9,13}$ que los carcinomas de mama de tipo luminal A y B, siendo estas diferencias estadísticamente significativas. La ausencia de asociación estadísticamente significativa observada en nuestro estudio entre los fenotipos HER2 y basal y la afec- tación de ganglio centinela se debe a que en estos pacientes con tumores más agresivos se llegó al diagnóstico de afectación ganglionar de manera preoperatoria y se realizó directamente linfadenectomía, sin necesidad de efectuar la técnica de ganglio centinela. Observamos asociación estadísticamente significativa entre los fenotipos moleculares y el estadio ganglionar. Los carcinomas de mama de tipo HER2 representan el segundo subtipo con mayor afectación ganglionar y tienen, después de los carcinomas de mama de tipo basal, la incidencia más alta en tumores indiferenciados. La mayor parte de los carcinomas de mama de tipo HER2 presentaba estadios avanzados en el momento del diagnóstico, de manera que el $51,8 \%$ se encontraba en estadio II y el $22,2 \%$ en estadio III. El índice de proliferación con Ki-67 en la mayor parte de los carcinomas de mama de tipo HER2 era, de forma significativa, moderado o alto (63\%) y un porcentaje alto de este subtipo de tumores expresaba p53 $(66,6 \%)$, pero sin diferencias estadísticamente significativas. HER2 es un importante marcador predictivo de respuesta a trastuzumab y lapatinib en cáncer de mama. Múltiples estudios han probado la eficacia y seguridad de trastuzumab en el tratamiento neoadyuvante, adyuvante y en la enfermedad metastásica de pacientes con cáncer de mama de tipo HER2.

En cuanto a los carcinomas de mama de tipo basal, la mayor parte de los mismos en nuestro estudio presenta un tamaño tumoral mayor de $2 \mathrm{~cm}$ en el momento del diagnóstico (únicamente el 22,7\% tenía menos de $2 \mathrm{~cm}$ ) y un estadio avanzado de la enfermedad, con un $81,8 \%$ en estadio II o III. Son tumores fundamentalmente indiferenciados (70\% de grado III) con mayor afectación ganglionar $(54,6 \%)$ que los carcinomas de tipo luminal y los carcinomas de tipo HER2. Según diversos autores, aunque los carcinomas de mama de tipo basal son generalmente de alto grado, no guardan relación con el factor pronóstico más importante en el cáncer de mama, la invasión ganglionar ${ }^{11,14,15}$. En nuestro estudio el $45,4 \%$ de los carcinomas de mama de fenotipo basal no presentaba compromiso 
ganglionar en comparación con el 57,5\% en el trabajo de Spitale y col $^{16}$, el $59 \%$ en el de Bauer y $\operatorname{col}^{17}$ y el $54,2 \%$ en el de Yang y col${ }^{18}$. Según concluyen estos autores, el mal pronóstico de este subtipo molecular no parece relacionarse con su capacidad metastásica intrínseca, sino con las características de proliferación y de apoptosis del tumor. De hecho, en nuestro estudio los carcinomas de mama de tipo basal se asocian de forma significativa a elevados índices de proliferación $(\mathrm{p}<0,001)$ observándose que el $68 \%$ presentaba un Ki-67 moderado o alto. El antígeno Ki-67, que identifica las células proliferantes dentro de un tumor, es una proteína nuclear que se encuentra en todas las fases del ciclo celular (G1, S, G2 y M) salvo en G0. Por tanto, cuanto mayor es su presencia, más agresivo es el tumor. Asimismo, aunque de forma no significativa $(\mathrm{p}=0,45)$, los carcinomas de mama de fenotipo basal se asociaron en un $72,7 \%$ a la expresión de p53. La mutación de p53 se considera un factor de mal pronóstico porque determina pérdida de la función supresora, activación del crecimiento celular y aumento del riesgo de progresión de la neoplasia.

Los estudios inmunohistoquímicos del cáncer de mama revelan la expresión de determinadas proteínas en las células tumorales por lo que se podrían considerar un reflejo válido de los estudios de biología molecular así como una alternativa válida a los costosos estudios genéticos. Aunque algunos autores han demostrado que los carcinomas de mama se pueden catalogar en subtipos similares a los de expresión génica mediante un panel de marcadores inmunohistoquímicos, en el momento actual no existe acuerdo sobre qué marcadores deben utilizarse en la clasificación del cáncer de mama, especialmente al definir los carcinomas de mama de fenotipo basal, ni sobre el porcentaje o intensidad de tinción celular requerido ${ }^{4,5}$. En el año 2004, Nielsen y $\mathrm{col}^{4}$ utilizando un panel de cuatro anticuerpos (RE, HER2, HER1 y CK5/6) observaron que existía una buena correlación entre el perfil génico y el perfil inmunohistoquímico. En este estudio, la sensibilidad de la IHQ en la clasificación de estos tumo- res fue de un $76 \%$ y la especificidad de un $100 \%$. Otros autores proponen la utilización de otros marcadores en la definición de los carcinomas de mama (CK14, ki-67, CK17, EGFR, c-kit, p63, P-cadherina y actina de músculo liso $)^{6,14,19}$. Para evitar sesgos en futuros ensayos clínicos y valorar su efectividad predictiva sería conveniente que los anatomopatólogos unificaran criterios en la determinación IHQ de los marcadores para la clasificación del cáncer de mama.

En la definición de carcinoma de mama de fenotipo basal, nosotros utilizamos los criterios aceptados por la mayor parte de autores. Consideramos carcinomas de mama de fenotipo basal aquellos casos negativos tanto para RH como HER2 (por tanto, eran carcinomas de mama triplenegativos -CMTN-) que además expresaran algún marcador basal. Como marcadores basales, además de los sugeridos por Rakha y col $^{14}$ (CK5/6 y/o CK14) añadimos la expresión de EGFR y/o p63 utilizados por otros autores ${ }^{6,19}$. Los términos basal y triple-negativo se utilizan en ocasiones como sinónimos pero, aunque comparten características clínicas y pronósticas, representan grupos poblacionales y técnicas de diagnóstico diferentes. No todos los carcinomas de mama definidos mediante IHQ como triple-negativos son de tipo basal cuando se analizan a través de pruebas de ADN. En el estudio de Rakha y col $^{20}$ sólo el $55,7 \%$ de los casos considerados como triple-negativo por IHQ (157 de los 282) fue de fenotipo basal cuando se agregó la expresión de CK5, CK6 o CK14. En otros artículos ${ }^{21}$ el $85 \%$ de los CMTN se categorizó como carcinomas de fenotipo basal. Nuestros resultados confirman que no todos los casos triple-negativos son de tipo basal, pues no expresan marcadores de células basales. Con el panel de cuatro marcadores inmunohistoquímicos detectamos una alta frecuencia de carcinomas de tipo basal entre los triple-negativos (22/26 carcinomas, $84,6 \%$ ), muy parecida a la encontrada por Livasy y $\mathrm{col}^{21}$ (85\%) y superior a la frecuencia encontrada por Rakha y $\operatorname{col}^{20}(55,7 \%)$. La mayor parte de los carcinomas de mama de tipo basal en nuestro estudio mostró un fenotipo basal/mioepitelial incompleto. En 
el $18,2 \%$ de los casos se detectó la expresión de los cuatro marcadores, en $22,7 \%$ de los casos la expresión de tres de los cuatro marcadores, en $45,4 \%$ la expresión de dos de los cuatro marcadores y en $13,7 \%$ la expresión de un único marcador. Esta variabilidad en los porcentajes de positividad con los diferentes anticuerpos utilizados justifica la necesidad de usar un panel conjunto (no sólo un anticuerpo) con el objetivo de obtener una mayor sensibilidad. El marcador IHQ más sensible fue EGFR, que se expresó en el 76,2\% de las pacientes con carcinoma de mama de tipo basal, seguido por CK5/6 que se expresó en el 57,1\%. En nuestro estudio, al igual que en muchos estudios previos ${ }^{16-18}$ el subtipo basal es el que presenta la menor tasa de supervivencia y el menor tiempo de recaída lo cual viene a significar que probablemente tenemos la posibilidad de acotar un subgrupo de peor pronóstico simplemente utilizando marcadores inmunohistoquímicos que forman parte de la rutina normal del estudio de cáncer de mama en la mayoría de los servicios de Anatomía de Patológica.

Diversos estudios han demostrado que dentro de los subtipos histológicos existe importante variación pronóstica, de forma que ciertos tipos como el tubular, mucinoso, medular y adenoide quístico tienen buen pronóstico mientras que otros como los carcinosarcomas, carcinomas de mama en anillo de sello o los carcinomas inflamatorios muestran mal pronóstico ${ }^{22}$. Sin embargo, estos subtipos constituyen un porcentaje pequeño del total de carcinomas de mama y, de hecho, a pesar de esta diversidad en el comportamiento, el $70-80 \%$ de los tumores se clasifican como carcinomas ductales infiltrantes. Resulta evidente que bajo esa misma denominación se están clasificando tumores con un comportamiento clínico diferente. De hecho, cuando en nuestro estudio analizamos las diferencias pronósticas en los tres grandes subgrupos histológicos (CDI, CLI y otros) no se observaron diferencias estadísticamente significativas en cuanto al riesgo de recaída ni de supervivencia. Sin embargo, cuando utilizamos la clasificación molecular del cáncer de mama, los resultados revelaron diferencias pronósticas estadísticamente significativas, observándose un mayor riesgo de recaída y peor supervivencia en los subtipos basal y HER2. Las diferencias pronósticas observadas en nuestro estudio son comparables con estudios realizados en otros países y tienen importantes implicaciones. Nuestros resultados indican que la aplicación de estos marcadores IHQ en el ámbito clínico permite obtener una mejor definición pronóstica del cáncer de mama y diseñar los tratamientos de forma individualizada para obtener el mayor beneficio con el menor riesgo posible. Es indudable la importancia de evaluar el riesgo individual de cada paciente y de identificar a aquellas pacientes que tienen mayor agresividad tumoral, que pueden recibir tratamiento en una fase temprana del diagnóstico, así como aquellas pacientes con tumores de menor patogenicidad que no requieren someterse a los riesgos y efectos secundarios que implican los tratamientos citotóxicos. Conocer el pronóstico de un paciente con carcinoma de mama permite, por una parte, informar al paciente y/o a sus familiares sobre la posible evolución de la enfermedad y, por otra, permite elegir el tratamiento más apropiado para cada paciente.

\section{BIBLIOGRAFÍA}

1. Perou CM, Sørlie T, Eisen MB, van de Rijn M, JeFFREY SS, REES CA et al. Molecular portraits of human breast tumors. Nature 2000; 406: 747752.

2. Sørlie T, Tibshirani R, Parker J, Hastie T, Marron JS, Nobel A et al. Repeated observation of breast tumor subtypes in independent gene expression data sets. Proc Natl Acad Sci U S A. 2003 ; 100: 8418-8423.

3. Foekens JA, Atkins D, Zhang Y, Sweep FC, HaRBECK N, PARADISO A et al. Multicenter validation of a gene expression-based prognostic signature in lymph node-negative primary breast cancer. J Clin Oncol 2006; 24: 1665-1671.

4. Nielsen TO, Hsu FD, Jensen K, Cheang M, Karaca G, Hu Z et al. Immunohistochemical and clinical characterization of the basal-like subtype of invasive breast carcinoma. Clin Cancer Res 2004; 10: 5367-5374. 
5. Livasy CA, Karaca G, Nanda R, Tretiakova MS, Olopade OI, Moore DT et al. Phenotypic evaluation of the basal-like subtype of invasive breast carcinoma. Mod Pathol 2006; 19: 264271.

6. Burness ML, Grushro T, Olopade OI. Epidermal Growth Factor Receptor in triple-negative and basal-like breast cancer: promising clinical target or only a marker? Cancer 2010; 16 : 23-32.

7. Blows FM, Driver KE, Schmidt MK, Broeks A, van LeEuwen FE, Wesseling J et al. Subtyping of breast cancer by immunohistochemistry to investigate a relationship between subtype and short and long term survival: a collaborative analysis of data for 10,159 cases from 12 studies. PLoS Med 2010; 7: e1000279.

8. Tsakountakis N, Sanidas E, Stathopoulos E. Correlation of breast cancer risk factors with HER 2/NEU protein overexpression according to menopausal and estrogen receptor status. BMC Women Health 2005; 5: 1-9.

9. Huang W, Newman B, Millikan R. Risk of breast cancer according to the status of HER-2/neu oncogen amplification. Cancer Epidemiol Biomarkers Prev 2000; 9: 65-71.

10. Rouzier R, Perou CM, Symmans WF, Ibrahim N, Cristofanilli M, Anderson K et al. Breast cancer molecular subtypes respond differently to preoperative chemotherapy. Clin Cancer Res. 2005; 11: 5678-5685.

11. Carey la, Perou CM, Livasy CA, Dressler lG, Cowan D, Conway K et al. Race, breast cancer subtypes, and survival in the Carolina Breast Cancer Study. JAMA 2006; 295: 2492-2502.

12. Loi S, Haibe-Kains B, Desmedt C, Lallemand F, TutT AM et al. Definition of clinically distinct molecular subtypes in estrogen receptorpositive breast carcinomas through genomic grade. J Clin Oncol 2007; 25: 1239-1246.

13. Kreike B, van Kouwenhove M, Horlings H, Weigelt B, Peterse H, Bartelink $\mathrm{H}$ et al. Gene expression profiling and histopathological characterization of triple-negative/basal-like breast carcinomas. Breast Cancer Res. 2007; 9: R65.

14. Rakha EA, Putti TC, Abd El-Rehim DM Paish C, Green AR, Powe DG, Lee AH et al. Morpholo- gical and immunophenotypic analysis of breast carcinomas with basal and myoepithelial differentiation. J Pathol 2006; 208: 495-506.

15. Da Silva L, Clarke C, LaKhani SR. Demystifying basal-like breast carcinomas. J Clin Pathol 2007; 60: 1328-1332. Review.

16. Spitale A, Mazzola P, Soldini D, Mazzuccheldi L, BORDONI A. Breast cancer classification according to immunohistochemical markers: clinicopathologic features and short-term survival analysis in a population-based study from the South of Switzerland. Ann Oncol 2009; 20: 628-635.

17. Bauer KR, Brown M, Cress RD, Parise CA, CAGGIANO V. Descriptive analysis of estrogen receptor (ER)-negative, progesterone receptor (PR)-negative, and HER2-negative invasive breast cancer, the so-called triple-negative phenotype: a population-based study from the California cancer Registry. Cancer 2007; 109: 1721-1728.

18. Yang XR, Sherman ME, Rimm DL, LissowsKa J, Brinton LA, Peplonska B et al. Differences in risk factors for breast cancer molecular subtypes in a population-based study. Cancer Epidemiol Biomarkers Prev 2007; 16: 439-443.

19. Matos I, Dufloth R, Alvarenga M, Zeferino LC, Schмiтt F. p63, cytokeratin 5, and P-cadherin: three molecular markers to distinguish basal phenotype in breast carcinomas. Virchows Arch 2005; 447: 688-694.

20. RaKha EA, El-Sayed ME, Green AR, LeE AH, RoBERTSON JF, Ellis IO. Prognostic markers in triple-negative breast cancer. Cancer 2007; 109: 25-32.

21. Livasy CA, Karaca G, Nanda R, Tretiakova MS, Olopade OI, Moore DT et al. Phenotypic evaluation of the basal-like subtype of invasive breast carcinoma. Mod Pathol 2006; 19: 264271.

22. Ellis IO, Galea M, Broughton N, Locker A, BlaMEy RW, Elston CW. Pathological prognostic factors in breast cancer. II. Histological type. Relationship with survival in a large study with long-term follow-up. Histopathology 1992; 20: 479-489. 